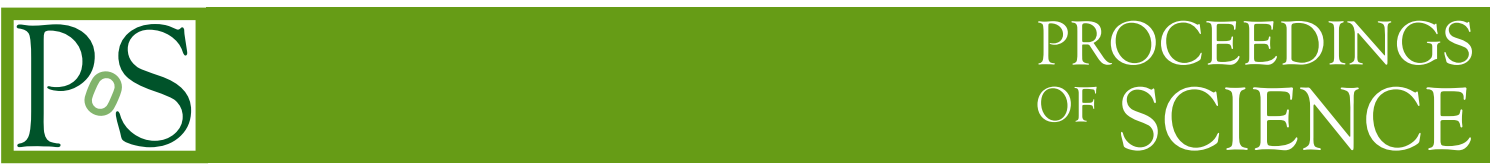

\title{
Astrophysics Potential of Hyper-Kamiokande
}

\author{
Luis Labarga ${ }^{* t}$ \\ University Autonoma Madrid \\ E-mail: luis. labarga@uam.es \\ on behalf of The Hyper-Kamiokande proto-Collaboration
}

Hyper-Kamiokande is a next generation underground water Cherenkov detector based on the highly successful Super-Kamiokande experiment. It will be capable of observing - far beyond the sensitivity of the Super-Kamiokande detector - proton decay, atmospheric neutrinos, and neutrinos from astronomical sources. It will also serve as a far detector, $295 \mathrm{~km}$ away, of a long baseline neutrino experiment for the upgraded J-PARC beam.

Further to the detector planned size of more than one order of magnitude larger than predecessor experiments, its improved photon yield will enable superior signal efficiency and background rejection. This is particularly relevant for astrophysical neutrinos, such as the supernova burst neutrinos, supernova relic neutrinos, and solar neutrinos, allowing a much more precise study of their physics phenomena. This poster presents the performance of the current detector design using MC simulations and dedicated event-reconstruction tools and the corresponding potential expected for neutrino astrophysics.

38th International Conference on High Energy Physics

3-10 August 2016

Chicago, USA

\footnotetext{
*Speaker.

${ }^{\dagger}$ supported by EU H2020-MSCA-RISE-2014-GA641540, SKPLUS.
} 
On the strength of a double Nobel prize winning experiment (Super)Kamiokande and an extremely successful long baseline neutrino programe, the third generation Water Cherenkov detector, Hyper-Kamiokande (Hyper-K, HK), is being developed by an international collaboration as a leading worldwide experiment based in Japan [1]. It will be the largest underground water Cherenkov detector in the world and will be instrumented with new technology photosensors (PMTs), faster and with higher quantum efficiency than the ones in Super-Kamiokande [2]. The currently existing accelerator in J-PARC will be steadily upgraded to reach a MW beam by the start of the experiment. Figure 1 pictures relevant details of HK.

Hyper-K will be a multipurpose detector addressing some of the most significant questions facing particle physicists today [2] through a multi-decade physics programe that will start in the middle of the next decade. HK aims to measure a CP-violating phase in the leptonic sector, as well as refine the neutrino mixing angles and mass squared difference parameters. HK will prove Grand Unification in at least one order of magnitude further than the current World best limits (by Super-Kamiokande). Hyper-K will observe an unprecedented number of neutrino events should a supernova occurs within galaxy distances, thus providing the much needed experimental results to understand the mechanism of the explosion and to shed light on neutrino interactions at very high temperatures [4]. The ability of measuring the energy spectrum of the neutrino relics of all supernova along the history of the Universe, will give us a deep, unique insight, on the later.

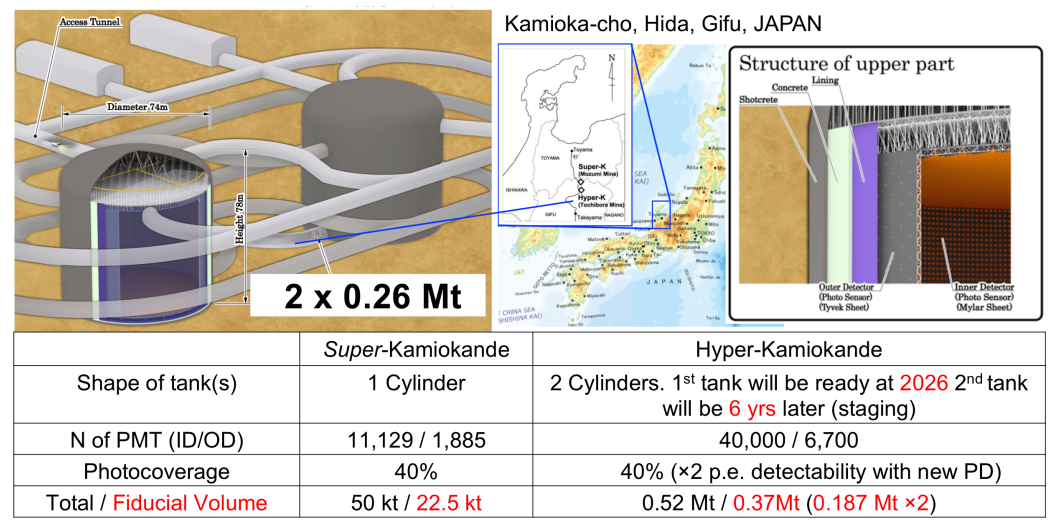

Figure 1: The Hyper-Kamiokande project. The table compares relevant features with its predecesor, the currently active Super-Kamiokande experiment.

\section{Solar Neutrinos}

We have estimated expected sensitivities from solar $v$ measurements in 10 years of Hyper-K observation based on the current Super-K knowledge. Even though the increase of the spallation background level in Hyper-K will be up to a factor of 2.7 as compared to Super-K beacuse of its shallower site, the much greater size and better energy resolution result on a significant reduction of the uncertainties. The sensitivity of the identification of the neutrino oscillation parameters between solar and reactor neutrinos by day-night asymmetry is estimated to $5.1 \sigma$ (Fig. 2-left). The possibility of spectrum upturn observation is estimated to $4.9 \sigma$ level (Fig. 2-middle). The solar hep neutrino could be measured with a few Mton year data (Fig. 2-right).

\section{Supernova Neutrinos}

The number of neutrino events measured by Hyper-Kamiokande from a galactic supernova is illustrated by the table at Fig. 3-left-bottom. They will be enough to provide detailed information 

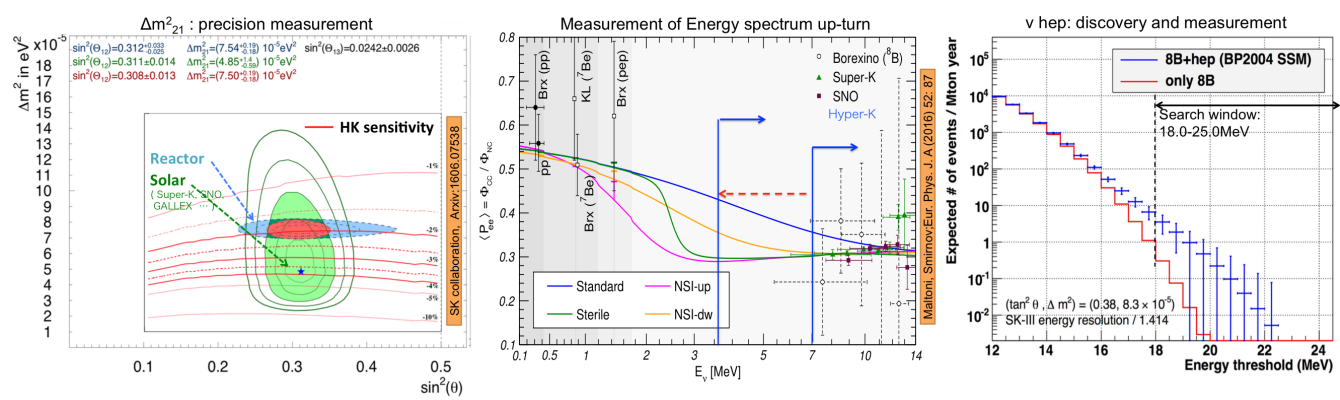

Figure 2: Left: Allowed neutrino oscillation parameter region from all the solar $v$ experiments (green), reactor $v$ from KamLAND (blue) and combined (red) from one to five sigma lines and three sigma filled area. The star shows the best fit parameter from the solar neutrinos. The contour of the expected day-night asymmetry with $6.5 \mathrm{MeV}$ energy threshold is overlaid. Middle: Impact of new physics on solar $v$ survival probability [3]. Right: Expected solar $v$ fluxes (with $v$ oscillation) in HK. The horizontal axis is energy threshold in electron total energy and the vertical axis is expected event rate in the energy range from the threshold up to $25 \mathrm{MeV}$. BP2004 SSM fluxes are assumed. The effect of background events, reduction efficiencies and systematic uncertainties are not considered.

about the time profile and the energy spectrum for inspecting supernova explosion mechanisms (Fig. 3-middle) and to gain significant insight on fundamental neutrino physics, namely the neutrino mass, the mass hierarchy and the neutrino oscillations in the supernova (Fig. 3-left-top). All these analyses benefit very much from the good energy resolution, lower energy threshold and large photo-coverage of HK.
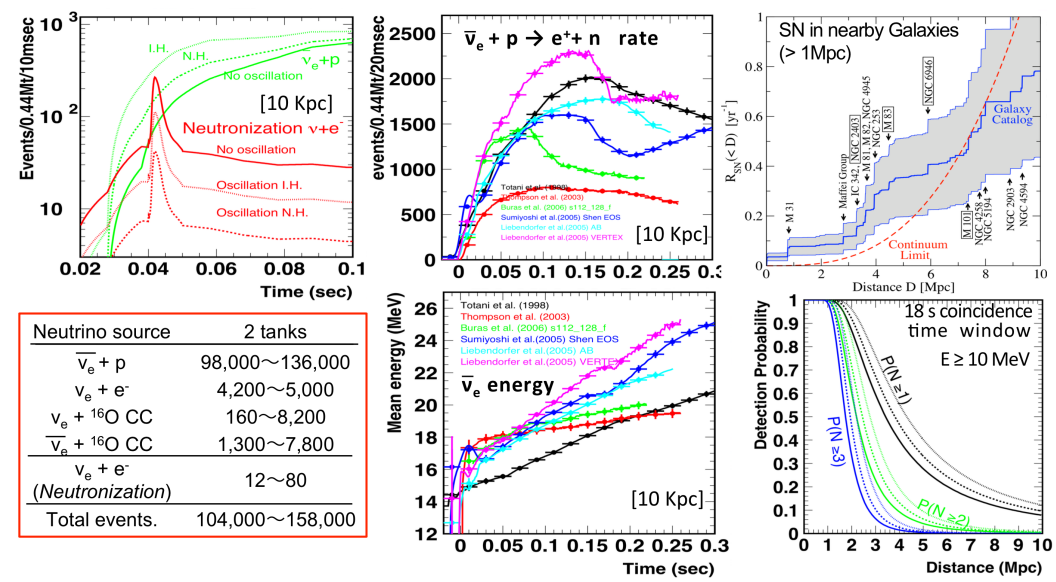

Figure 3: Left-top: Expected event rate at the time of neutronization burst for a supernova at $10 \mathrm{kpc}$. Leftbottom: Summary table of expected supernova neutrino events. Middle: Inverse beta event rate (top) and mean energy of $\bar{v}_{e}$ (bottom) predicted by supernova simulations [5] for the first 0.3 seconds after the onset of a $10 \mathrm{kpc}$ distant burst. Right: Cumulative calculated supernova rate versus distance (top, taken from [6]). (bottom) Detection probability of supernova $v$ versus distance at Hyper-K; $\mathrm{N}$ denotes the number of events per burst required. Solid, dotted, and dashed curves are for $v$ oscillation scenarios of no oscillation, N.H., and I.H., respectively.

The cumulative supernova rate versus distance as estimated in [6] is shown in Fig. 3-righttop. If Hyper-K could see signals out to $4 \mathrm{Mpc}$ then we expect a supernova about every three years. Figure 3-right-bottom shows the corresponding detection probability by HK. 


\section{Diffuse Supernova Neutrino Background or Supernova Relic Neutrino (SRN)}

It is estimated that $10^{17}$ supernova explosions have occurred over the entire history of the universe. The neutrinos produced there must fill the present universe and their flux is estimated to be a few tens $/ \mathrm{cm}^{2} / \mathrm{sec}$. Figure 4-left shows the SRN spectra predicted by various models. Figure 4-right shows the expected observation of the SRN spectrum in the Hyper-K detector in the configuration of a Gd doping at $0.1 \%$. The expected number of SRN events in the energy range of 10-30 MeV is about 560 with 10 years of live time with 2 tanks. It important to measure down to $\sim 10 \mathrm{MeV}$ in order to explore the history of supernova bursts back to the epoch of red shift $(z) \sim 1$.
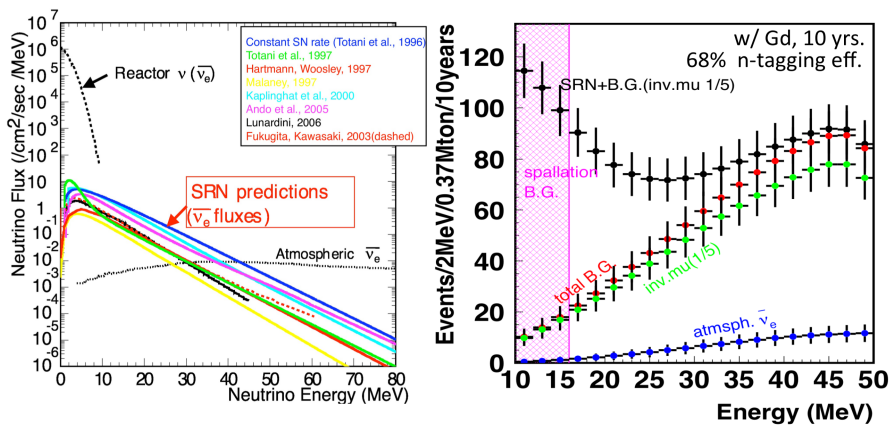

Figure 4: Left: Predictions for SRN spectrum. Fluxes of reactor and atmospheric $v, \mathrm{~s}$ are also shown. Right: Expected spectrum of the SRN signals at Hyper-K with 10 years of live-time. The black dots show signal + background (red dots). Green and blue show background contributions from the invisible $\mu$ and $v_{e}$ components of atmospheric neutrinos. A SRN flux prediction [7] was used, and a 67\% detection efficiency of $8 \mathrm{MeV} \gamma$ cascades and a factor of 5 reduction in the invisible- $\mu$ background were assumed.

Epilogue. Hyper-Kamiokande is the next generation Megaton-like, neutrino and nucleon decay experiment. Remarkably, HK will provide by far the most complete experimental neutrino astrophysics program of the next decades.

\section{References}

[1] Hyper-Kamiokande proto-Collab.; K. Abe et al., KEK Preprint 2016-21, ICRR-Report 701-2016-1

[2] Y. Nishimura, New $50 \mathrm{~cm}$ Photo-Detectors for HK; Talk at this Conference; Contribution \#638 M. Gonin, HK's Neutrino Oscillation Physics Sensitivity; Talk at this Conference; Contribution \#1038 L. Labarga, non-Accelerator physics with HK; Talk at this Conference; Contribution \#516

[3] M. Maltoni, A.Y. Smirnov; Solar neutrinos and neutrino physics, Eur. Phys. J. A (2016) 5287.

[4] H. Duan, G. M. Fuller, J. Carlson, and Y.-Z. Qian, Phys.Rev. D74, 105014 (2006)

H. Duan, G. M. Fuller, J. Carlson, and Y.-Z. Qian, Phys.Rev.Lett. 97, 241101 (2006)

B. Dasgupta, A. Dighe, G. Raffelt, and A. Y. Smirnov, Phys. Rev. Lett. 103, 051105 (2009)

A. Friedland, Phys.Rev.Lett. 104, 191102 (2010)

H. Duan and A. Friedland, Phys.Rev.Lett. 106, 091101 (2011)

[5] T. Totani, K. Sato, H. E. Dalhed, and J. R. Wilson, Astrophys. J. 496, 216 (1998)

T. A. Thompson, A. Burrows, and P. A. Pinto, Astrophys. J. 592, 434 (2003)

R. Buras, H.-T. Janka, M. Rampp, and K. Kifonidis, Astron. Astrophys. 457, 281 (2006)

K. Sumiyoshi et al., Astrophys. J. 629, 922 (2005)

[6] S. Ando, J. F. Beacom, and H. Yu üksel; Phys. Rev. Lett. 95, 171101 (2005)

[7] K. Sumiyoshi et al.; Astrophys. J. 629, 922 (2005) 